\title{
Child Poverty in Rural America: New Data Shows Increases in 41 States
}

WILLIAM P. O'HARE AND SARAH SAVAGE

$\mathrm{O}$ n August 29th, the U.S. Census Bureau released new data on child poverty that show a disturbing increase in rural child poverty rates in many states. The child poverty rate is the most widely used indicator of child well-being because poverty is closely linked to undesirable outcomes in areas such as health, education, emotional welfare, and delinquency. Changes in child poverty signal important changes in children's quality of life and life chances.

Table 1 shows how child poverty rates have changed between 2000 and 2005 for the children living outside metropolitan areas in each state. (Data are collected in 2000 and 2005, but they actually reflect poverty in 1999 and 2004.) Release of data from the Census Bureau's new American Community Survey provide the first chance we have had to look at state-by-state changes in rural child poverty since the 2000 Census data provided rural child poverty data for the 1990 to 2000 period.

The data shown here are based on the official poverty measure as determined by the U.S. Office of Management and Budget. The official poverty measure consists of a series of income thresholds based on family size and composition. The 2005 poverty line was $\$ 19,806$ for a family of two adults and two children.

\section{A UTHORS}

Dr.William P. O'Hare (bbohare@comcast.net) is a visiting senior fellow at the Carsey Institute.

Sarah Savage (ssavage@cisunix.unh.edu) is a research assistant at the Carsey Institute.

\section{Key results include:}

- Rural child poverty rates increased between 2000 and 2005 in forty-one of the fifty states (data were not available in four states).

- In eighteen states, the increase in the rural child poverty rate was higher than the increase in the overall U.S. rural child poverty rates between 2000 and 2005.

- The state with the biggest percentage point increase in rural child poverty between 2000 and 2005 was Maine (+ 7.4 percentage points), followed by North Carolina ( +6.7 percentage points), and Mississippi (+ 6.1 percentage points).

- Only six states showed a decrease in the rural child poverty rate, lead by Wyoming with a 4.2 percentage point decrease.

- Five states (Maine, North Carolina, Mississippi, Ohio and Indiana) experienced increases of 5 percentage points or more in the rural child poverty rate between 2000 and 2005 .

- The rural child poverty rate in 2005 ranges from a low of just over 6 percent in Connecticut to a high of nearly 37 percent in Mississippi.

- Five states (Mississippi, Louisiana, New Mexico, Arizona, and Alabama) all had rural child poverty rates above 30 percent in 2005, which reflects the pervasive child poverty in the rural South.

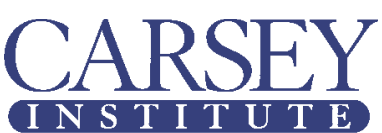

Building KNOWLEDGE FOR FAmilies AND Communities IN THE 21ST CENTURY
Huddleston Hall, 73 Main Street, Durham, NH 03824

(603) 862-2821 • www.carseyinstitute.unh.edu

The Carsey Institute Reports on Rural America are supported by the Annie E. Casey Foundation's initiative to strengthen rural families, the Ford Foundation, and the W.K. Kellogg Foundation. 
Table 1. Child poverty in non-metropolitan areas ${ }^{1}$ by state: 2000 and 2005

\begin{tabular}{|c|c|c|c|c|c|c|c|}
\hline & \multicolumn{3}{|c|}{2000 Census } & \multicolumn{3}{|c|}{2005 American Community Survey } & \multirow[b]{2}{*}{$\begin{array}{c}\text { Percentage point } \\
\text { change in poverty rate } \\
\text { from } 2000 \text { to } 2005\end{array}$} \\
\hline & $\begin{array}{c}\text { Population under } \\
\text { age } 18^{*}\end{array}$ & $\begin{array}{l}\text { Population under } \\
\text { age } 18 \\
\text { below poverty }\end{array}$ & $\begin{array}{l}\text { Percent of population } \\
\text { under age } 18 \\
\text { below poverty }\end{array}$ & $\begin{array}{l}\text { Population under } \\
\text { age } 18^{*}\end{array}$ & $\begin{array}{l}\text { Population under } \\
\text { age } 18 \\
\text { below poverty }\end{array}$ & $\begin{array}{l}\text { Percent of population } \\
\text { under age } 18 \\
\text { below poverty }\end{array}$ & \\
\hline U.S. & $13,742,721$ & $2,644,052$ & 19.2 & $11,336,476$ & $2,552,591$ & 22.5 & 3.3 \\
\hline Alabama & 332,749 & 87,110 & 26.2 & 305,855 & 92,525 & 30.3 & 4.1 \\
\hline Alaska & 112,840 & 15,180 & 13.5 & 63,005 & 11,281 & 17.9 & 4.5 \\
\hline Arizona & 167,417 & 49,343 & 29.5 & 171,709 & 52,966 & 30.8 & 1.4 \\
\hline Arkansas & 331,938 & 81,708 & 24.6 & 267,433 & 78,474 & 29.3 & 4.7 \\
\hline California & 282,892 & 62,521 & 22.1 & 176,776 & 36,399 & 20.6 & -1.5 \\
\hline Colorado & 167,164 & 24,106 & 14.4 & 150,298 & 23,964 & 15.9 & 1.5 \\
\hline Connecticut & 34,966 & 2,336 & 6.7 & 67,820 & 4,270 & 6.3 & -0.4 \\
\hline Delaware & 34,417 & 5,273 & 15.3 & 36,240 & 6,595 & 18.2 & 2.9 \\
\hline District of Columbia & N/A & $\mathrm{N} / \mathrm{A}$ & $\mathrm{N} / \mathrm{A}$ & N/A & $\mathrm{N} / \mathrm{A}$ & $\mathrm{N} / \mathrm{A}$ & $\mathrm{N} / \mathrm{A}$ \\
\hline Florida & 241,825 & 55,002 & 22.7 & 224,488 & 54,076 & 24.1 & 1.3 \\
\hline Georgia & 651,551 & 150,267 & 23.1 & 420,562 & 115,467 & 27.5 & 4.4 \\
\hline Hawaii & 84,919 & 14,387 & 16.9 & 89,365 & 14,304 & 16.0 & -0.9 \\
\hline Idaho & 220,968 & 34,694 & 15.7 & 125,259 & 24,631 & 19.7 & 4.0 \\
\hline Illinois & 437,147 & 64,090 & 14.7 & 369,445 & 68,454 & 18.5 & 3.9 \\
\hline Indiana & 428,843 & 49,635 & 11.6 & 343,648 & 58,494 & 17.0 & 5.4 \\
\hline lowa & 391,775 & 43,773 & 11.2 & 291,437 & 40,536 & 13.9 & 2.7 \\
\hline Kansas & 297,659 & 42,243 & 14.2 & 234,632 & 41,680 & 17.8 & 3.6 \\
\hline Kentucky & 498,717 & 127,783 & 25.6 & 400,978 & 116,053 & 28.9 & 3.3 \\
\hline Louisiana & 296,626 & 92,517 & 31.2 & 281,915 & 88,837 & 31.5 & 0.3 \\
\hline Maine & 188,129 & 28,321 & 15.1 & 108,732 & 24,410 & 22.4 & 7.4 \\
\hline Maryland & 91,722 & 13,636 & 14.9 & 65,515 & 11,159 & 17.0 & 2.2 \\
\hline Massachusetts & 54,791 & 5,654 & 10.3 & $\mathrm{~N} / \mathrm{A}$ & $\mathrm{N} / \mathrm{A}$ & $\mathrm{N} / \mathrm{A}$ & $\mathrm{N} / \mathrm{A}$ \\
\hline Michigan & 426,474 & 57,527 & 13.5 & 413,720 & 73,203 & 17.7 & 4.2 \\
\hline Minnesota & 365,389 & 41,266 & 11.3 & 309,143 & 41,452 & 13.4 & 2.1 \\
\hline Mississippi & 491,699 & 150,376 & 30.6 & 417,931 & 153,366 & 36.7 & 6.1 \\
\hline Missouri & 437,843 & 88,848 & 20.3 & 339,942 & 83,511 & 24.6 & 4.3 \\
\hline Montana & 150,910 & 30,806 & 20.4 & 131,011 & 27,475 & 21.0 & 0.6 \\
\hline Nebraska & 209,175 & 28,292 & 13.5 & 176,518 & 27,184 & 15.4 & 1.9 \\
\hline Nevada & 65,920 & 8,101 & 12.3 & 60,330 & 9,414 & 15.6 & 3.3 \\
\hline New Hampshire & 115,543 & 10,126 & 8.8 & 102,684 & 10,408 & 10.1 & 1.4 \\
\hline New Jersey & N/A & N/A & N/A & $\mathrm{N} / \mathrm{A}$ & N/A & $\mathrm{N} / \mathrm{A}$ & $\mathrm{N} / \mathrm{A}$ \\
\hline New Mexico & 229,836 & 68,609 & 29.9 & 172,450 & 53,217 & 30.9 & 1.0 \\
\hline New York & 350,537 & 60,687 & 17.3 & 334,081 & 61,107 & 18.3 & 1.0 \\
\hline North Carolina & 620,149 & 128,003 & 20.6 & 631,052 & 172,761 & 27.4 & 6.7 \\
\hline North Dakota & 90,858 & 15,228 & 16.8 & 71,923 & 9,868 & 13.7 & -3.0 \\
\hline Ohio & 545,599 & 73,234 & 13.4 & 527,004 & 100,002 & 19.0 & 5.6 \\
\hline Oklahoma & 336,924 & 77,161 & 22.9 & 291,120 & 72,117 & 24.8 & 1.9 \\
\hline Oregon & 221,997 & 39,533 & 17.8 & 180,715 & 39,541 & 21.9 & 4.1 \\
\hline Pennsylvania & 433,571 & 65,103 & 15.0 & 416,020 & 74,612 & 17.9 & 2.9 \\
\hline Rhode Island & 13,897 & 1,668 & 12.0 & $\mathrm{~N} / \mathrm{A}$ & $\mathrm{N} / \mathrm{A}$ & $\mathrm{N} / \mathrm{A}$ & $\mathrm{N} / \mathrm{A}$ \\
\hline South Carolina & 304,110 & 72,489 & 23.8 & 247,714 & 65,833 & 26.6 & 2.7 \\
\hline South Dakota & 130,019 & 26,332 & 20.3 & 100,750 & 19,833 & 19.7 & -0.6 \\
\hline Tennessee & 426,258 & 83,870 & 19.7 & 354,727 & 86,580 & 24.4 & 4.7 \\
\hline Texas & 827,383 & 205,766 & 24.9 & 726,242 & 206,081 & 28.4 & 3.5 \\
\hline Utah & 170,771 & 22,891 & 13.4 & 80,364 & 11,196 & 13.9 & 0.5 \\
\hline Vermont & 105,163 & 13,244 & 12.6 & 86,217 & 12,912 & 15.0 & 2.4 \\
\hline Virginia & 334,054 & 56,893 & 17.0 & 227,005 & 46,541 & 20.5 & 3.5 \\
\hline Washington & 250,389 & 49,332 & 19.7 & 168,512 & 34,747 & 20.6 & 0.9 \\
\hline West Virginia & 227,053 & 62,977 & 27.7 & 164,114 & 45,996 & 28.0 & 0.3 \\
\hline Wisconsin & 423,362 & 43,238 & 10.2 & 329,572 & 41,164 & 12.5 & 2.3 \\
\hline Wyoming & 88,783 & 12,873 & 14.5 & 75,480 & 7,801 & 10.3 & -4.2 \\
\hline
\end{tabular}

$\mathrm{N} / \mathrm{A}=$ Not applicable.

1. Metropolitan areas are defined based on the the U.S. Census Bureau's definitions as of June 1999. The full list of counties and New England towns used to define metropolitan areas is available online at: http://www.census.gov/population/estimates/metro-city/99mfips.txt.

${ }^{*}$ for whom poverty is determined 